\title{
DIFFUSION FLAME ATTACHMENT AND LIFT-OFF IN THE NEAR WAKE OF A FUEL INJECTOR
}

\author{
E. FERNÁNDEZ, ${ }^{2}$ V. KURDYUMOV ${ }^{1,2}$ AND A. LIÑÁN ${ }^{1}$ \\ ${ }^{1}$ E.T.S.I. Aeronáuticos \\ UPM, Madrid, Spain \\ ${ }^{2}$ Instituto Nacional de Técnica Aerospacial \\ Madrid, Spain
}

\begin{abstract}
An analysis is presented for the flow, temperature, and concentration fields in the region of attachment of fuel jet diffusion flames, near the wake of the fuel injector, where upstream heat conduction and diffusion are important. The characteristic scales for the size and velocity in this region are identified as $l_{\mathrm{N}}=$ $\sqrt{v_{0} / A}$ and $U_{\mathrm{N}}=\sqrt{v_{0} A}$, in terms of the kinematic viscosity of the fuel and the wall value of the fuel velocity gradient. The parameters that characterize the structure of the flame attachment region are identified, and some representative cases are numerically analyzed. There are cases with large activation energy, for which the flame will be attached if the Karlovitz number, $v_{0} A / U_{\mathrm{L}}^{2}$, or non-dimensional velocity gradient, is smaller than a critical value; for larger values, the flame lifts off far from the rim of the injector. For smaller values of the activation energy, the diffusion flame is attached, with its edge near the rim of the injector if the Karlovitz number is small; the distance to the injector of the edge of the flame grows with the Karlovitz number, and the edge takes on a triple-flame structure.
\end{abstract}

\section{Introduction}

In gaseous jet diffusion flames, the flame may be either attached to the near-wake region of the injector or lifted off, away from the injector, if the velocity of the fuel jet or that of the coflowing air stream goes above a critical value.

The problem of determining the conditions of attachment of diffusion flames and flame lift-off has received considerable attention in the literature. See, for example, work by Robson and Wilson [1], Kawamura and Asato [2], and Kawamura et al. [3], and in particular, the extensive reviews by Takahashi and Schmoll [4] and Takahashi et al. [5]. However, due to the difficulties in numerical or analytical description of the flow in the region of flame attachment, and the large number of parameters involved, there are few reliable general descriptions of the flame and flow field in this region.

The Reynolds number of the flow associated with jet diffusion flames is typically large compared with unity and is often large enough for the flow to be turbulent. Due to this, the mixing between the fuel stream and the stagnant or coflowing air takes place in a thin mixing layer between the streams, without significant effects from upstream diffusion or conduction. These effects are important only in a small region, which we call the Navier-Stokes region, near the rim of the injector. Upstream conduction and diffusion plays an essential role in the attachment of the diffusion flame to the injector, as was already understood by Gaydon and Wolfhard [6], who indicated that the molecular mixing in the region creates a small volume of combustible mixture able to sustain a premixed flame propagating against the flow.

The formulation of the problem of the flow structure in the region was advanced by Liñán [7]. Simplified models for the flow in the region were given by Wichman [8-9] and Buckmaster and Weber [10]. Reliable numerical simulations based on the complete equations were given by Takahashi et al. [5].

Here, the formulation of Liñán [7] has been generalized and completed with the appropriate description of the boundary conditions. The main scales and non-dimensional parameters characterizing the flame attachment process have been identified. The problem has been numerically solved for a few representative cases in which the thickness of the injector wall at the rim is small compared with the characteristic size of the region.

Although triple-deck concepts have to be used to describe the flow at the end of a splitter plate separating two streams, here, the analysis has been confined to the small Navier-Stokes region, at the core of the triple deck (Fig. 1). The first descriptions of the flow in the Navier-Stokes region, lying at the end of the injector where the two boundary layers begin to merge, were given by Dijkstra [11] and Daniels [12]. The flow is determined there by the two wall velocity gradients, $A$ for the fuel flow and $\alpha A$ for the air flow, at the end of the injector wall. As shown by Higuera and Liñán [13], these values, if $\alpha \neq 0$, are modified by overpressures created by displacement effects due to heat release from their boundary layer values upstream of the triple-deck region where 


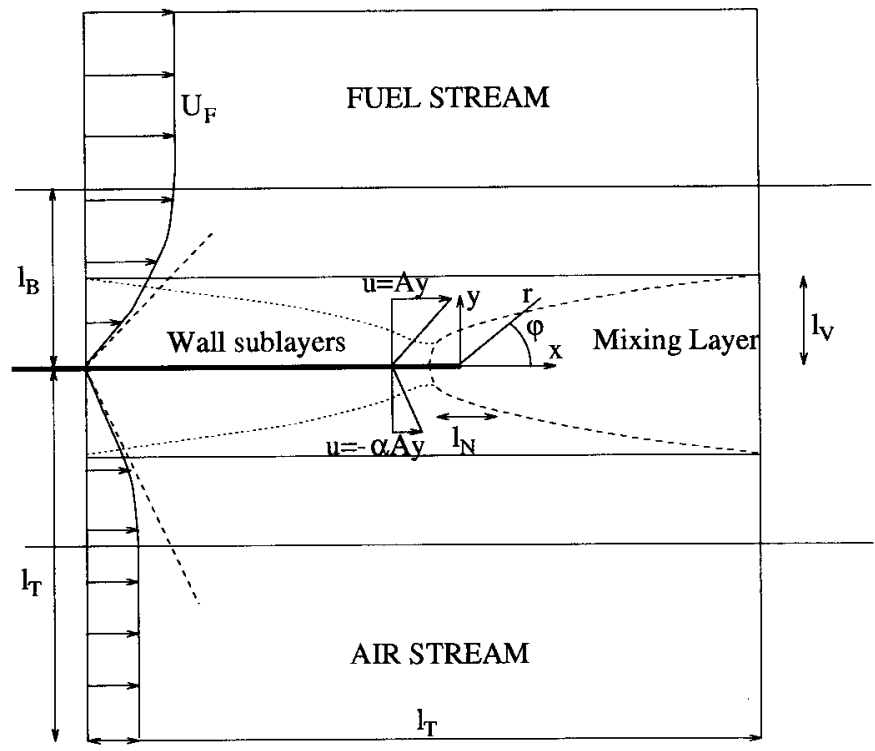

FIG. 1. Sketch of the coordinate system and the characteristic scales of the triple-deck and NavierStokes regions: $l_{\mathrm{T}}=l_{\mathrm{B}} R e_{\mathrm{B}}^{1 / 4}$, $l_{\mathrm{V}}=l_{\mathrm{B}} R e_{\mathrm{B}}^{-1 / 4}, l_{\mathrm{N}}=l_{\mathrm{B}} R e_{\mathrm{B}}^{-1 / 2}$. these overpressures act. The fuel wall velocity gradient, $A$, gives the characteristic timescale $1 / A$ for flow in the Navier-Stokes region. The length and velocity scales in the region are $l_{\mathrm{N}}=\sqrt{v_{0} / A}$ and $U_{\mathrm{N}}=$ $\sqrt{v_{0} A}$, based on the kinematic viscosity of the fuel. They are such that the local Reynolds number is of the order unity, and therefore, upstream heat conduction and diffusion effects are important. In terms of the Reynolds number, $R e_{\mathrm{B}}=U_{\mathrm{F}} l_{\mathrm{B}} / v_{0}$, based on the velocity $U_{\mathrm{F}}$ of the fuel stream and the thickness, $l_{\mathrm{B}}$, of the fuel boundary layer, $l_{\mathrm{N}} / l_{\mathrm{B}}=U_{\mathrm{N}} / U_{\mathrm{F}} \sim$ $R e_{\mathrm{B}}^{-1 / 2}$, while $l_{\mathrm{T}}=l_{\mathrm{B}} R e_{\mathrm{B}}^{1 / 4}$. Thus, one can understand why Takahashi et al. [5] needed to use a large computational domain to incorporate effects of the overpressures in the triple-deck region.

Important parameters characterizing the structure of the flow in the flame attachment region are the ratio, of the wall velocity gradients, $\alpha$, the non-dimensional thickness, $h$, of the injector wall, measured with $l_{\mathrm{N}}$, and the Damköhler number $\left(U_{\mathrm{L}} /\right.$ $\left.U_{\mathrm{N}}\right)^{2}$, or its inverse, the Karlovitz number $v_{0} A / U_{\mathrm{L}}^{2}$, based on the premixed flame propagation velocity, $U_{\mathrm{L}}$, of the stoichiometric mixture between the fuel and air. An analysis of the effects of the heat release due to diffusion flames on the flow in the wake of injectors with moderately large values of $h$ was given by Higuera and Liñán [13].

Here, we consider the flow in the Navier-Stokes region to be laminar, quasi-steady, and quasi-twodimensional, but we can conjecture that the results will also apply, at least qualitatively, to turbulent flows. In these flows, the scales of the flame attachment region are the friction velocity and the thickness of the viscous sublayer, where the local Reynolds number is of the order unity and the Reynolds stresses are no longer dominant; the main effect of turbulence is to introduce time variations in the wall velocity gradients.

Gravity effects have not been included in the formulation. The effects of gravity appear first in the analysis of the reacting mixing layer of the tripledeck region, when the velocity due to the buoyancy forces, of the order of $\left(g l_{\mathrm{T}}\right)^{1 / 2}$ in this layer, becomes of the order of the characteristic forced flow velocity, $U_{\mathrm{F}} R e_{\mathrm{B}}^{-1 / 4}$, in the layer. If the jet is directed upward, a vertical acceleration of the reacting mixture due to gravity may lead to entrainment into the mixing layer, which increases the value of the velocity gradient, $A$, that determines the structure of the NavierStokes region. For gravity to affect directly the structure of the Navier-Stokes region, $\left(g l_{\mathrm{N}}\right)^{1 / 2}$ must be of the order of $U_{\mathrm{N}}$.

\section{Formulation}

With the scale $l_{\mathrm{N}}$ of the Navier-Stokes region, we see the base of the fuel and the air boundary layers as two parallel streams of uniform shear, $A$ and $\alpha A$, approaching the end of the injector that appears as a splitter plate, with the plate at temperature $T_{0}$. They begin to mix and react when they reach the Navier-Stokes region.

The reaction is modeled, for simplicity of the presentation, by a single, irreversible, one-step reaction, $\mathrm{F}+s \mathrm{O}_{2} \rightarrow(1+s) \mathrm{P}+(Q)$, where $s$ grams of oxygen is consumed and $(1+s)$ grams of product is generated, together with a thermal energy $Q$ per unit gram of fuel consumed. The fuel consumption rate per unit volume is modelled by an Arrhenius rate 


$$
W_{\mathrm{F}}=B \rho^{2} Y_{\mathrm{F}} Y_{\mathrm{O}} \exp (-E / R T)
$$

involving, respectively, the pre-exponential factor, the density, the mass fractions of fuel and oxygen, the activation temperature $E / R$, and the temperature.

In the analysis given here, we consider the Prandtl number, $\mathrm{Pr}$, to be constant, as well as the Lewis numbers $L e_{\mathrm{F}}$ and $L e_{\mathrm{O}}$ of fuel and oxygen, and the molecular mass of the mixture. We use a power law dependence of the coefficient of viscosity with $T$ as $\mu / \mu_{0}=\left(T / T_{0}\right)^{m}$.

The equations describing the steady two-dimensional flow in the Navier-Stokes region are written using $l_{\mathrm{N}}=\sqrt{v_{0} / A}$ and $U_{\mathrm{N}}=\sqrt{v_{0} A}$ as scales for the spatial coordinates, and velocity $\vec{v}, \rho_{0} U_{\mathrm{N}}^{2}$ as scale for the pressure variations $p, \rho_{0}$ for the density, and $\mu_{0}$ for the viscosity. The initial values $Y_{\mathrm{F} 0}$ and $Y_{\mathrm{O} O}$ of the fuel and oxygen mass fractions are used as scales for $Y_{\mathrm{F}}$ and $Y_{\mathrm{O}}$. Then, the non-dimensional equations take the form

$$
\begin{gathered}
(\nabla \cdot \rho \vec{v})=0 \\
(\rho \vec{v} \cdot \nabla) \vec{v}=-\nabla p+\nabla \cdot \tau^{\prime} \\
\operatorname{Pr}(\rho \vec{v} \cdot \nabla \theta)=\nabla(\mu \nabla \theta)+(1+S) w \\
\operatorname{Pr}\left(\rho \vec{v} \cdot \nabla Y_{\mathrm{F}}\right)=\nabla\left(\mu \nabla Y_{\mathrm{F}}\right) / L e_{\mathrm{F}}-w \\
\operatorname{Pr}\left(\rho \vec{v} \cdot \nabla Y_{\mathrm{O}}\right)=\nabla\left(\mu \nabla Y_{\mathrm{O}}\right) / L e_{\mathrm{O}}-S w \\
\mu=(1+\gamma \theta)^{m}, \rho=(1+\gamma \theta)^{-1}
\end{gathered}
$$

Here, $\tau_{i j}^{\prime}=\mu\left(\nabla_{i} \cdot v_{j}+\nabla_{j} \cdot v_{i}\right)$ is the viscous stress tensor, and $\theta=\left(T-T_{0}\right) /\left(T_{s}-T_{0}\right)$ is the nondimensional temperature rise based on the initial value of the temperature $T_{0}$ and the adiabatic flame temperature $T_{\mathrm{s}}=T_{0}+Q Y_{\mathrm{Fo}} / c_{\mathrm{P}}(1+S)$ of the stoichiometric fuel air mixture; $\gamma=\left(T_{\mathrm{s}}-T_{0}\right) / T_{\mathrm{s}}$ is a heat release parameter, and $S=s Y_{\mathrm{F} 0} / Y_{\mathrm{OO}}$ is the mass of air required to burn the unit mass of the fuel stream.

The non-dimensional reaction rate, $w$, is given by

$$
w=d \beta^{3} \rho^{2} Y_{\mathrm{F}} Y_{\mathrm{O}} \exp \left(\frac{\beta(\theta-1)}{(1+\gamma(\theta-1) /(\gamma+1))}\right)
$$

where $\beta=E\left(T_{\mathrm{s}}-T_{0}\right) / R T_{\mathrm{s}}^{2}$ and $d=\beta^{-3}\left(B \rho_{0} Y_{\mathrm{O}}\right.$ $\operatorname{Pr} / A) \exp \left(-E / R T_{\mathrm{s}}\right)$ are the Zeldovich number and reduced Damköhler number, respectively. For single reaction schemes, the Damköhler number is a good characterization of the chemistry; however, for real schemes where we find a large number of reactions, the Damköhler number is not well defined. A better characterization of the chemistry for this case is given by the stoichiometric, premixed, planar laminar flame velocity, $U_{\mathrm{L}}$. For an Arrhenius reaction with a large activation energy, this velocity $U_{\mathrm{L}}$ was calculated by Zeldovich and Frank Kamenetskii [14], leading, in first approximation, to $U_{\mathrm{L}}^{2}=$ $2 B Y_{\mathrm{O} 0} \beta^{-3}\left(\lambda_{0} / c_{\mathrm{P}}\right) \exp \left(-E / R T_{\mathrm{s}}\right) /(1+\gamma)^{2-m}$. This can be used to write the reduced Damköhler number used above, in terms of the Karlovitz number, $K a=v_{0} \mathrm{~A} / U_{\mathrm{L}}^{2}$. Thus, $K a^{-1}=\left(U_{\mathrm{L}} / U_{\mathrm{N}}\right)^{2}=2 d / \operatorname{Pr}^{2}(1$ $+\gamma)^{2-m}$, which we anticipate to be of the order unity in the flame attachment regime.

We write the boundary conditions for equation 1 , the case of plate of thickness $h \ll 1$, as

$$
\begin{gathered}
\theta=u=v=\partial Y_{\mathrm{F}} / \partial y=\partial Y_{\mathrm{O}} / \partial y=0 \\
\text { at } y=0, x<0
\end{gathered}
$$

and

$$
\begin{aligned}
& r^{2}=x^{2}+y^{2} \rightarrow \infty \\
& y>0: u-y=v=\theta=Y_{\mathrm{F}}-1=Y_{\mathrm{O}}=0 \\
& y<0: u=\alpha y=v=\theta=Y_{\mathrm{F}}=Y_{\mathrm{O}}-1=0
\end{aligned}
$$

upstream and far from the plate, outside the downstream mixing layer. Notice that we do not allow for a finite $x$ displacement of the $u$ component of the velocity at large $r$. Downstream, for $x \rightarrow \infty$, the flow must approach the asymptotic solution of the boundary layer for equation 1 , to be described below.

The modification of equations 3 and 4 to account for the finite non-dimensional thickness, $h$, of the injector wall is straightforward. The generalization of this formulation to more realistic reaction mechanisms is also straightforward.

\section{Asymptotic Form of the Solution}

The decay toward zero, for $r \gg 1$, of the velocity perturbations is so weak that it must be determined, as shown below, to arrive at an accurate numerical solution of the problem.

\section{Asymptotic Solution in the Wake Mixing Layer}

Downstream, the thickness of the mixing layer, of order $x^{1 / 3}$, becomes small compared with $x$, so that the conservation equations 1 can be approximated by their boundary layer form. In addition, the residence time becomes large compared with the reaction time, and thus, the flame can be considered, in first approximation, as an infinitely thin flame sheet corresponding to the Burke-Schumann limit of infinite reaction rates. For $x \gg 1$, the flow in the mixing layer becomes self-similar, as in the non-reacting case analyzed in Ref. [15]. The solution is of the form

$$
\begin{aligned}
\psi & =x^{2 / 3} F(\zeta), p=C x^{2 / 3}, Y=\Phi(\zeta) \\
\theta & =G(\zeta), x \gg 1, y \sim x^{1 / 3}
\end{aligned}
$$

where $\psi$ is the stream function, defined as $\partial \psi / \partial y=$ $\rho u,-\partial \psi / \partial x=\rho v$, and $\zeta=y / x^{1 / 3}$ is the similarity variable, of order unity in the mixing layer. Here, $C$ 


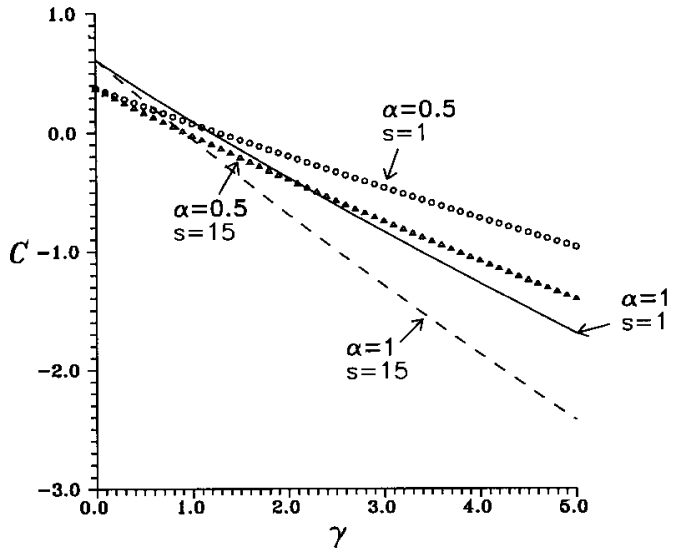

FIG. 2. Pressure gradient constant, $C$, as a function of $\gamma$.

is a constant, to be determined as part of the numerical solution, which measures the pressure gradient required to eliminate a finite $x$-wise velocity displacement, which we encounter further downstream in the triple-deck region, as shown in Ref. [13]. The functions $F, G$, and $\Phi$ of $\zeta$ are given by the solution, with boundary conditions derived from equation 4 , of a system of ordinary differential equations, which we do not include here for the sake of brevity. For large values of $|\zeta|, F$ behaves as

$$
\begin{aligned}
& F(\zeta \rightarrow+\infty)=\frac{1}{2} \zeta^{2}+C \\
& \text { and } F(\zeta \rightarrow-\infty)=-\frac{\alpha}{2} \zeta^{2}-\frac{C}{\alpha}
\end{aligned}
$$

where the constant $C(\alpha, \gamma, S)$ also determines the fuel and air entrainment velocities, $v_{\mathrm{e}}=(2 / 3)$ $C x^{-1 / 3}$ and $(2 / 3 \alpha) C x^{-1 / 3}$, by the mixing layer, and therefore the flow in the outer inviscid region.

The most important result from the analysis of the Hakkinen and Rott mixing layer is the value of the constant $C$. When $C$ is positive, which is the case for low values of $\gamma$, the mixing layer entrains fuel and air from outside. As shown in Fig. 2, for values of $\gamma$ larger than a critical value $\gamma_{\mathrm{c}}(\alpha, S)$, due to the effect of heat release, $C$ becomes negative, and the mixing layer introduces an outward displacement on the flow. For $\alpha=S=1, C$ changes sign at $\gamma=1.19$. For $\gamma=0$, the equation for $F$ is not coupled with the equations for $\Phi$ and $G$, and $C$ is a function only of $\alpha$.

In the limiting case $\alpha=0$, when there is no forced flow in the oxidizer side, the function $F(\zeta)$ tends, for $\zeta \rightarrow-\infty$, to a constant, $F_{\infty}$. In this case, $C=0$, and the pressure gradient in the mixing layer is absent. At small values of $\alpha$, the constant $C$ is linear with $\alpha$, $C=-F_{\infty} \alpha$. The value of $F_{\infty}(\gamma, S)$ determines the air entrainment velocity by the mixing layer, $v_{\mathrm{e}}=$ $(2 / 3) F_{\propto} x^{-1 / 3}$.

\section{Far-Field Inviscid Flow Perturbations}

The positive or negative values of the entrainment velocity generated by the reacting mixing layer dominate the velocity perturbations outside the NavierStokes region. Thus, it can be shown that far from the plate and outside the downstream mixing layer, the solution of the governing equations 1, matched appropriately with the downstream boundary layer and upstream boundary layers near the plate, is given by

$$
\begin{gathered}
\theta=0, \begin{array}{l}
Y_{\mathrm{F}}=1, \quad Y_{\mathrm{O}}=0, \quad y>0 \\
Y_{\mathrm{O}}=0, \quad Y_{\mathrm{F}}=1, \quad y<0
\end{array} \\
\psi= \begin{cases}y^{2} / 2+2 C r^{2 / 3} \sin (2(\pi-\varphi) / 3) / \\
\sqrt{3}+D \ln \left(y / r^{1 / 3}\right), & y>0 \\
-\alpha y^{2} / 2-(2 C / \alpha) r^{2 / 3} \sin (2(\pi+\varphi) & \\
/ 3) / \sqrt{3}-D \alpha^{-4 / 3} \ln \left(-y / r^{1 / 3}\right), & y<0\end{cases}
\end{gathered}
$$

This flow field corresponds to an isothermal flow, with $\omega \rightarrow-1+D y^{-2}$ at $y \rightarrow \infty$ and $\omega \rightarrow \alpha-$ $D \alpha^{-4 / 3} y^{-2}$ at $y \rightarrow-\infty$, where $\omega=\partial v / \partial x-\partial u / \partial y$. $D$ is determined by matching with the solution for the upstream viscous boundary layers described below.

\section{Upstream Viscous Layers}

As was shown in Ref. [11], viscous effects must be retained in the wall viscous sublayers for $(-x) \gg 1$, shown in Fig. 1. There, $\theta=0$ and

$$
\psi=\left\{\begin{array}{lr}
y^{2} / 2+D \Phi\left(y /(-x)^{1 / 3}\right), & y>0 \\
-\alpha y^{2} / 2-D \alpha^{-4 / 3} \Phi\left((-y) /(-x)^{1 / 3}\right), & y<0
\end{array}\right.
$$

where $\Phi(z)$ is given by $3 \Phi^{\prime \prime \prime}=z^{2} \Phi+1$, with the conditions $\Phi(0)=\Phi^{\prime}(0)=\Phi^{\prime \prime}(\infty)=0$. The solution leads to $\Phi^{\prime}(\infty)=1.15 \ldots$, which when used with the matching conditions with the outer inviscid solution, determines $D=4 C / 3^{3 / 2} \Phi^{\prime}(\infty)$, where $\Phi^{\prime}(\infty)$ $=-1.15 \ldots$.

\section{Numerical Results}

With the above-mentioned boundary conditions, we can proceed with numerical methods to solve the system of equation 1 . The parameters remaining in the formulation are $\alpha, \gamma, S, U_{\mathrm{L}}^{2} / U_{\mathrm{N}}^{2}, \beta, \operatorname{Pr}, L_{\mathrm{F}}, L_{\mathrm{O}}$, and $h$. In the calculations presented here, only for $h$ $=0$ and a few representative cases, we fix $\mathrm{Pr}=$ $0.72, L e_{\mathrm{F}}=L e_{\mathrm{O}}=1, \alpha=S=1$, and $m=0.5$.

We begin by showing in Fig. 3 how the total nondimensional heat transfer to the plate, $q$, based on $\lambda_{0}\left(T_{\mathrm{s}}-T_{0}\right)$, varies with the Damköhler number, $1 / K a$, and the non-dimensional activation energy. The calculations for the case $\gamma=0$, when the effects 


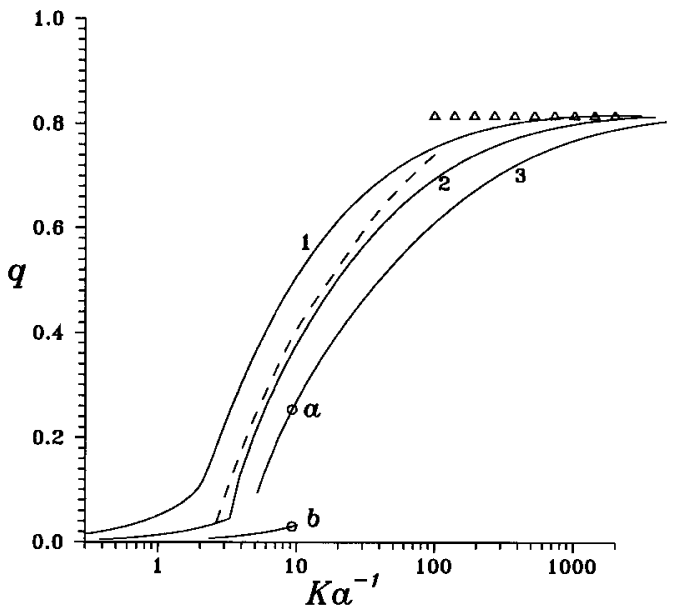

FIG. 3. Total heat transfer to the injector as a function of inverse Karlovitz number (lines 1, 2, 3: $\gamma=0, \beta=8$, 10, 12; dashed line: $\gamma=5, \beta=12$; triangles: Burke-Schumann limit, $\gamma=0$ ). $S=\alpha=1$.

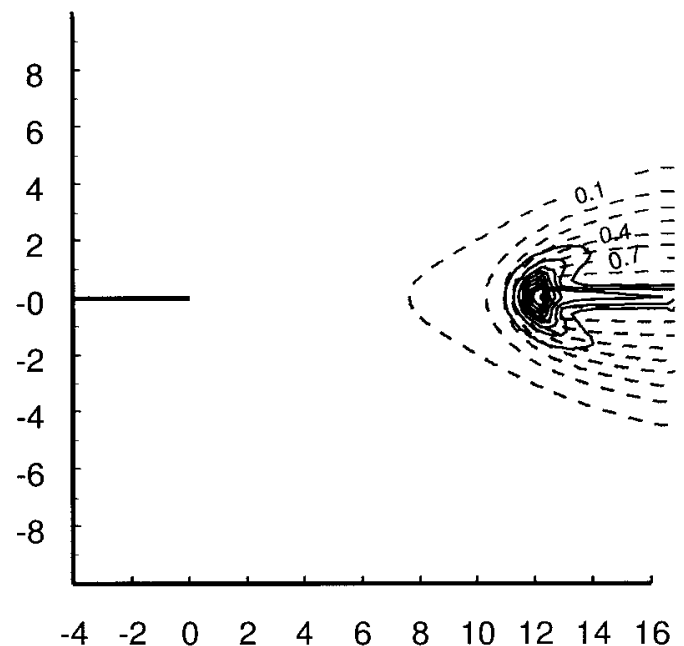

FIG. 4. Temperature (dashed lines) and reaction rate (solid lines) for frozen solution, $\gamma=0, S=1, \beta=12$, and $d=0.26\left(K a^{-1}=9.26\right)$.

of the thermal expansion on the flow field are left out, show that for values of $\beta<10$, only one solution exists, with a smooth transition from frozen flow to diffusion-controlled combustion at large Damköhler numbers. In these solutions, we find a nearly frozen regime upstream of the edge of the diffusion flame; this edge moves downstream with decreasing values of the Damköhler number, becoming a triple flame, as shown in Fig. 4, which approaches the splitter plate at large Damköhler numbers. The position of the triple flame is a result of the balance of the fluid

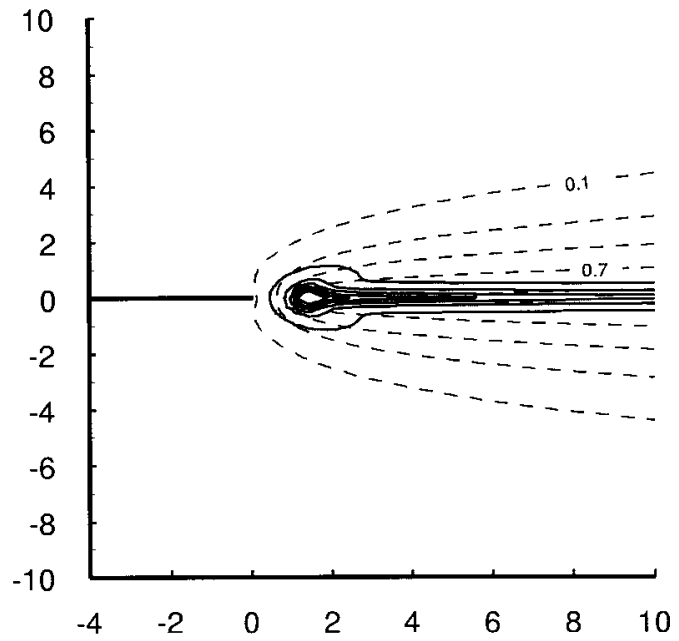

FIG. 5. Temperature (dashed lines) and reaction rate (solid lines) for attached solution, $\gamma=0, S=1, \beta=10$, and $d=0.8\left(K a^{-1}=3.08\right)$.

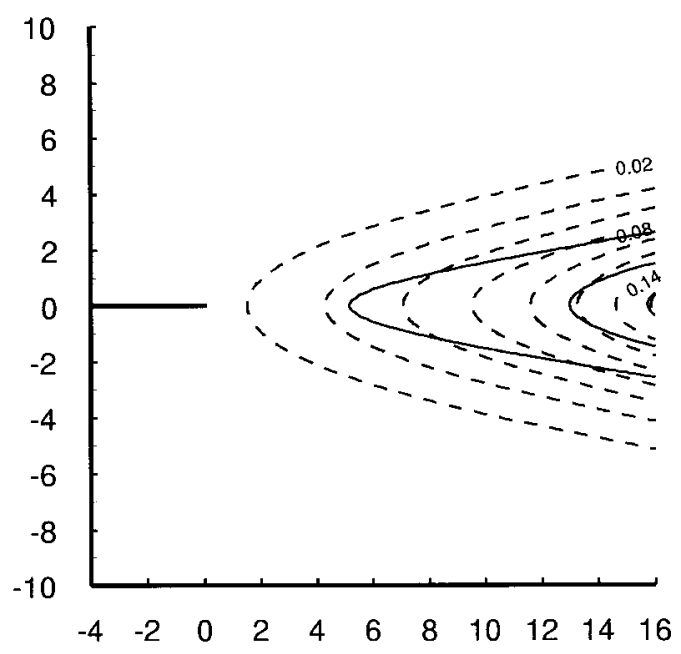

FIG. 6. Temperature (dashed lines) and reaction rate (solid lines) for attached solution, $\gamma=0, S=1, \beta=12$, and $d=0.24\left(K a^{-1}=9.26\right)$.

flow and triple-flame front velocity, which decreases with the ratio of the thickness of the mixing layer. This front velocity decreases to zero if the flame lies close to the injector. For $\beta>10$, three solutions exist for Karlovitz numbers lower than a critical value $K a_{\text {c }}$ (the intermediate one is unstable and cannot be obtained by our numerical procedure). One of the solutions shows, as seen in Fig. 5, a flame attached to the Navier-Stokes region. The other corresponds, as seen in figure 6 , to a weakly reacting flow in the region. 


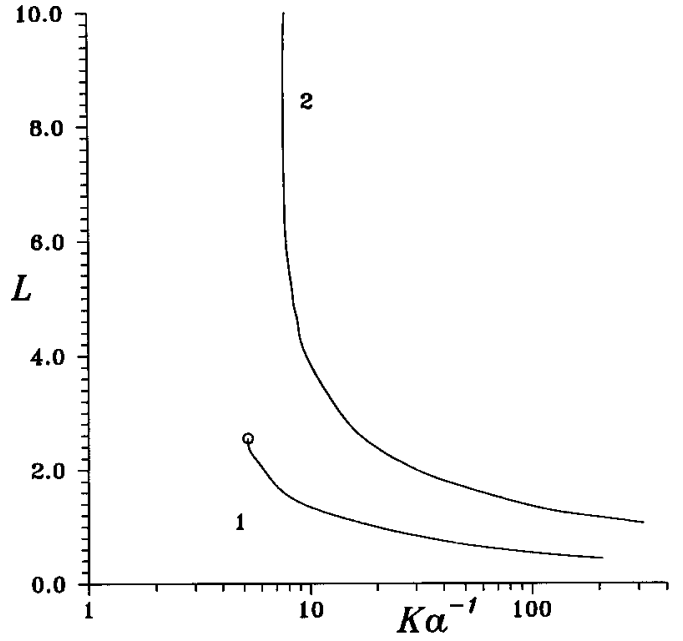

FIG. 7. Distance from the edge of the flame to the injector as a function of the inverse Karlovitz number (line 1: $\gamma=0, \beta=12$; line $2: \gamma=5, \beta=12$ ).

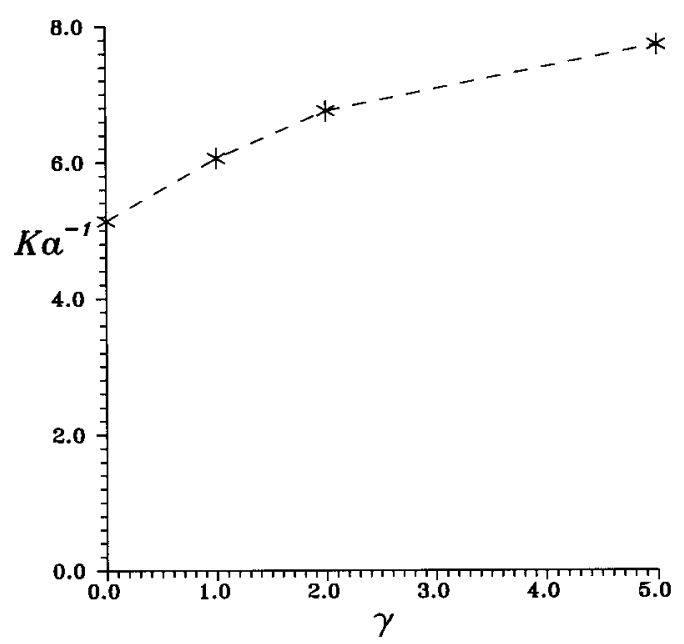

FIG. 8. Critical values for lift-off of the Damköhler number or the inverse of the Karlovitz number, as a function of $\gamma$ for $\beta=12$.

If, for the attached flame, we define the flame edge as the point with maximum reaction rate, its non-dimensional distance, $L$, to the injector, shown in Fig. 7, increases with decreasing values of the Damköhler number, until, at $K a^{-1}=K a_{\mathrm{c}}^{-1}$, we find a sudden jump in $q$ to a very small value corresponding to the lifted flame. The dependence of $L$ on the Damköhler number is weak for large values of $\mathrm{Ka}^{-1}$.

The critical Damköhler number, $K a_{\mathrm{c}}^{-1}$, for lift-off, when the attached flame solution suddenly jumps into the frozen solution, is shown as a function of $\gamma$ in Fig. 8.

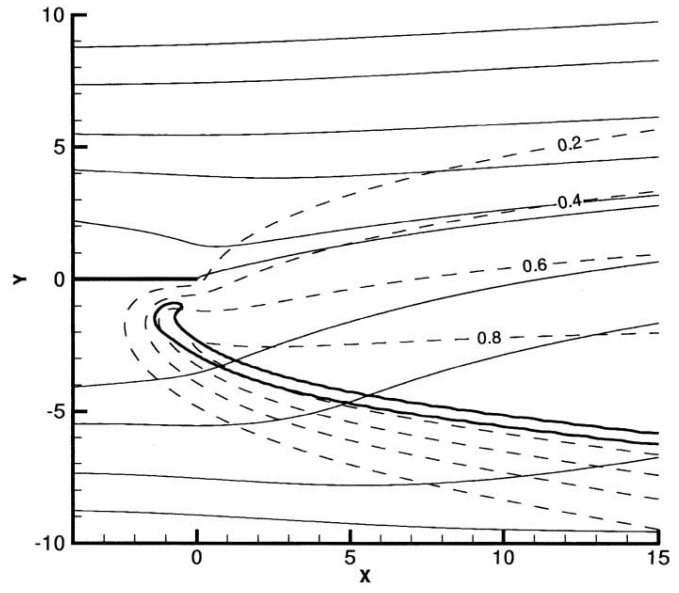

FIG. 9. Stream function (thin solid lines), isolines for the temperature (dashed lines), and reaction rate (thick solid line) for an attached flame, $\gamma=5, S=5, \beta=10$, and $d$ $=2000\left(K a^{-1}=525\right)$.

In Figs. 5 and 6, corresponding to the points $a$ and $b$ in Fig. 3, we show the isotherms and reaction rate isolines. The difference between the forms of the attached solution and the nearly frozen solution is self-explanatory. The form of the diffusion flame edge depends, as shown by Dold et al. [16] and Daou and Liñán [17], on the ratio of the thickness of the mixing layer and the thickness of the planar stoichiometric flame. Only for large values of this ratio is the flame edge a triple flame, as shown in Fig. 4. Near lift-off, the ratio is of order unity and the leading edge of the flame appears to be what Takahashi et al. [5], called a reaction center.

As representative of the form of the flame edge for a typical hydrocarbon fuel, $\gamma \sim 5$ and $S \sim 15$, we give in Fig. 9 the flow field and shape of the flame. Due to the large value of the mass stoichiometric air/fuel ratio, $S$, the flame lies well on the oxidizer side.

\section{Conclusions}

We have presented in this work the theoretical basis for the analysis of the structure of the region of flame attachment of diffusion flames to the rim of an injector. We have identified the characteristic values for the velocity, $U_{\mathrm{N}}=\sqrt{\alpha A}$, and size, $l_{\mathrm{N}}=$ $\sqrt{\alpha / A}$, of the region of diffusion flame attachment to the rim of a fuel injector, in terms of the values $A$ and $\alpha A$ of the uniform shear of the flows of the fuel and air surrounding the region. We have shown how to describe the asymptotic form of the velocity perturbations to these flows, at large distances compared with $l_{\mathrm{N}}$. These perturbations are independent of the kinetics of the reaction as long as the flame 
remains attached, and therefore the flame is diffusion controlled in the downstream mixing layer.

The calculated perturbations have been used in the numerical description of the structure of the flame attachment region, which we present here only for some representative cases. They show the role of the Damköhler number $\left(U_{\mathrm{L}} / U_{\mathrm{N}}\right)^{2}$ or of its inverse, the Karlovitz number, $v_{0} \mathrm{~A} / U_{\mathrm{L}}^{2}$, to characterize the flame attachment and flame lift-off.

The large number of parameters involved and the few cases analyzed numerically in this paper (only for infinitely thin injector walls) limit the comparison with the experimental results to qualitative aspects. For quantitative agreement, the calculations are being extended to finite values of $h$ and to more realistic kinetics.

Although there been extensive empirical research on the subject of flame attachment, a direct quantitative comparison is not possible due to the lack of sufficiently detailed fluid flow data to estimate the velocity gradient at the wall.

\section{Acknowledgments}

This work has been supported by the Instituto Nacional de Técnica Aeroespacial (INTA), under the Thermofluidynamics Programme number IGB4400903, and by the Spanish DCICYT, under Contract No. PB94-0040.

\section{REFERENCES}

1. Robson, K., and Wilson, M. J. G., Combust. Flame 12:626-634 (1969).

2. Kawamura, T., and Asato, K., in Proc. Second European Symp. on Combustion, The Combustion Institute, Pittsburgh, pp. 611-616, 1975.
3. Kawamura, T., Asato, K., and Mazaki, T., Combust. Sci. Technol. 22:211-216 (1980).

4. Takahashi, F., and Schmoll W. J., Proc. Combust. Inst. 23:677-683 (1990).

5. Takahashi, F., Schmoll, W. J., and Katta, V. R., Proc. Combust. Inst. 27:675-684 (1998).

6. Gaydon, A. G., and Wolfhard, H. G., Flames: Their Structure, Radiation, and Temperature, Chapman \& Hall, London, 1953.

7. Liñán, A., in Combustion in High Speed Flows (J. Buckmaster, T. L. Jackson, and A. Kuman, eds.), Kluwer Academic, Boston, 1994, pp. 461-476.

8. Wichman, I. S., Combust. Sci. Technol. 64:295-313 (1989).

9. Wichman, I. S., Combust. Flame 117:384-393 (1999).

10. Buckmaster, J., and Weber, R., Proc. Combust. Inst. 26:1143-1149 (1996).

11. Dijkstra, D., Ph.D. dissertation, University of Groningen, Nova Press, Groningen, the Netherlands, 1974.

12. Daniels, P. G., Q. J. Mech. Appl. Math 30:319-342 (1977).

13. Higuera, F. J., and Liñán, A., J. Fluid Mech. 329:389411 (1996).

14. Zeldovich, Ya. B., and Frank-Kamenetskii, D. A., Zhurnal Fizicheskoi Khimiji 12:100 (1938).

15. Hakkinen, R. J., and Rott, N., AIAA J. 3:1553-1554 (1965).

16. Dold, J. W., Hartley, L. J., and Green, D., Dynamical Issues in Combustion Theory, Vol. 32 (P. C. Fife, A. Liñán, and F. A. Williams, eds.), IMA Volumes in Mathematics and its Applications, Springer-Verlag, New York, 1991, pp. 107-126.

17. Daou, J., and Liñán, A., Combust. Theory Model. 2:449-477 (1998).

\section{COMMENTS}

Forman Williams, UCSD, USA. This is interesting work. What would be the effect of roughness of the plate? The analysis assumes a smooth plate.

Author's Reply. For the analysis to be applicable, the grain size must be small compared with the Navier-Stokes length, which becomes of the order of the premixed flame thickness near liftoff.

Andy C. McIntosh, University of Leeds, UK. A most interesting piece of work showing how Karlovitz number determines the location of the attachment of the diffusion flame behind the fuel/air splitter plate. Do the authors have any feel for the stability of the flame, both when it is close to the rim of the fuel injector, or when far away?

Author's Reply. The numerical results reported here are limited to equal diffusivity of the reactants when the structure is expected to be stable. Instabilities may be encountered when the Lewis number of the fuel is different from unity; even though the strong stretch effects on the flame edge have a stabilizing influence. Certainly, these instabilities may be more important near liftoff if the flame edge moves far away from the injector. 